\title{
Screw Tapper Device
}

National Cancer Institute

\section{Source}

National Cancer Institute. Screw Tapper Device. NCI Thesaurus. Code C50321.

A device designed to cut threads in a object 